\title{
$\beta$-fibrinogen gene $G / A-455$ polymorphism in relation to fibrinogen concentrations and ischaemic heart disease in Chinese patients with Type II diabetes
}

\author{
K.S.L. Lam ${ }^{1}$, O.C.K. Ma ${ }^{2}$, N.M.S. Wat ${ }^{1}$, L.C. Chan $^{3}$, E.D. Janus ${ }^{2}$ \\ ${ }^{1}$ Department of Medicine, University of Hong Kong, Queen Mary Hospital, Hong Kong \\ ${ }^{2}$ Clinical Biochemistry Unit, University of Hong Kong, Queen Mary Hospital, Hong Kong \\ ${ }^{3}$ Department of Pathology, University of Hong Kong, Queen Mary Hospital, Hong Kong
}

\section{Abstract}

Aims/hypothesis. We investigated the relation between the $G / A-455$ (Hae III) $\beta$-fibrinogen gene polymorphism and plasma fibrinogen concentration and its role in ischaemic heart disease in 264 Chinese patients with Type II (non-insulin-dependent) diabetes mellitus and 182 non-diabetic control subjects.

Methods. The G/A-455 polymorphism was determined in genomic DNA using polymerase chain reaction and Hae III restriction enzyme digestion. Fibrinogen was measured with the Claus method.

Results. Fibrinogen concentrations were higher in diabetic patients $(3.3 \pm 0.5$ vs $2.5 \pm 0.9 \mathrm{~g} / 1$ in controls, $p<0.0001)$ and in women $(p<0.03$ vs men). Allele frequency of the variant $A$ allele was $27 \%$ in both diabetic patients and control subjects' similar to findings in Caucasians. In control subjects, the $A A$ genotype was associated with higher fibrinogen concentrations $(2.8 \pm 0.38 \mathrm{~g} / \mathrm{l}$ vs $2.5 \pm 0.5$ in $G G$ or $G A$, $p<0.03$ ), contributing to $4 \%$ of the variance in plas- ma fibrinogen. The genotype effect was smaller and not significant among non-smokers, women and diabetic patients. Higher fibrinogen concentrations and $A A$ genotype frequency were found in diabetic patients with ischaemic heart disease $(p<0.05$ and $p<0.005$, respectively vs unaffected patients). In a multiple logistic regression model, $A A$ genotype, age and mean arterial pressure were associated with ischaemic heart disease, with odds ratios of 4.19 $(p<0.01), 1.05(p<0.0001)$ and $1.03(p<0.03)$, respectively.

Conclusion/interpretation. The G/A-455 polymorphism is a genetic determinant of fibrinogen concentrations and ischaemic heart disease in this Chinese cohort. It also interacts with environmental influences associated with smoking, the female sex and Type II diabetes in determining plasma fibrinogen concentrations. [Diabetologia (1999) 42: 1250-1253]

Keywords Fibrinogen, gene, polymorphism, Type II diabetes, ischaemic heart disease.
It has been increasingly recognised that the excess cardiovascular risk in Type II (non-insulin-dependent) diabetes mellitus could be partly contributed by an increased prothrombotic tendency, including increased fibrinogen concentrations [1], a strong pre-

Received: 28 December 1998 and in revised form: 15 April 1999

Corresponding author: Professor K.S. L. Lam,Department of Medicine, University of Hong Kong, Queen Mary Hospital, Hong Kong

Abbreviations: MAER, Mean albumin excretion rate; OR, odd's ratio. dictor of ischaemic heart disease and stroke in various large epidemiological studies [2]. Fibrinogen and fibrin accumulate in the atherosclerotic plaque at a rate proportional to the plasma fibrinogen concentration $[2,3]$. Genetic factors which affect the production or circulating concentrations of fibrinogen could therefore be causally related to the development of atherosclerosis.

Of the known fibrinogen gene polymorphisms, the $G / A-455$ polymorphism at the $5^{\prime}$-flanking region of the $\beta$-fibrinogen gene is the most important independent genetic determinant of plasma fibrinogen concentrations in Caucasian populations [4], with the highest concentrations found in subjects with the $A A$ 
genotype [4-7]. Its relation with plasma fibrinogen concentrations in Chinese has not been reported. In non-diabetic subjects, the presence of the less frequent $A$ allele was associated with more severe coronary artery disease, in univariate analysis, in the ECTIM study [3]. The $A A$ genotype was also more common in patients with cerebrovascular disease resulting from large-vessel disease in a study conducted in Germany [5]. There was a previous report of an association of the $G / A-455$ polymorphism with ischaemic heart disease in Type II diabetic patients but the association, surprisingly, was with the GG genotype instead [6].

In this study, we investigated the relation between the $\beta$-fibrinogen $G / A-455$ polymorphism and plasma fibrinogen concentrations in a Chinese cohort and attempted to explain its association with ischaemic heart disease in Type II diabetic patients.

\section{Subjects and methods}

Subjects. All subjects were unrelated southern Chinese with Type II diabetes $(n=264 ; 130$ men and 134 women, aged $52.4 \pm 16.2$ years, mean \pm SD). To provide an age-matched control group, data from 182 non-diabetic subjects were retrieved from the database of the Hong Kong Cardiovascular Risk Factor Prevalence Study [8] for comparative analysis. Studies were conducted after informed consent had been obtained from the subjects. Clinical data were obtained from the clinic records and confirmed by a personal interview, physical examination and an electrocardiogram carried out on the same day. Ischaemic heart disease was diagnosed by a history of myocardial infarction, angina, coronary bypass surgery or angioplasty, or evidence of myocardial infarction (pathogical Q-wave) or notable horizontal ST segment depression ( $\geq 2 \mathrm{~mm}$ ) in a 12-lead resting electrocardiogram (interpreted by N.M.S. Wat).

DNA analysis. DNA was extracted from leucocytes in 5-10 ml EDTA blood as reported [8]. A $1.3 \mathrm{~kb}$ portion of the $\beta$-fibrinogen gene, spanning from -1178 bp from the start of transcription to $+122 \mathrm{bp}$, was amplified by polymerase chain reaction, digested with Hae III, and the resultant fragments analysed as described [4-7].

Assays. Fibrinogen was measured by the Clauss method [6] on the Cobas Fibro (Roche Diagnostics, Nutley, N. J., USA) using fresh trisodium citrated samples. Fasting blood samples for $\mathrm{HbA}_{1 \mathrm{c}}$, creatinine, lipids and apolipoproteins were measured using standard methods as described [8]. Mean albumin excretion rate (MAER), assayed nephelometrically, was estimated from two consecutive 12 -h overnight urine collections.

Statistical analysis. Statistical analysis was done using the SPSS (version 4 Chicago, USA) statistics package. Values for fibrinogen and triglyceride, not normally distributed, were log-transformed before analysis. Data were processed using univariate (Student's $t$ test, Pearson's correlation analysis or chi-squares as appropriate) and multivariate analysis (stepwise multiple linear regression or multiple logistic regression). Duncan's multiple range test was used for comparing multiple groups. A $p$ value less than 0.05 was considered statistically significant (corrected for multiple comparison if indicated).
Table 1. Plasma fibrinogen $(\mathrm{g} / \mathrm{l})$ concentrations in control subjects and diabetic patients with different genotypes

\begin{tabular}{lrlll}
\hline & \multicolumn{1}{c}{$n$} & $G G$ & $G A$ & $A A$ \\
\hline Control subjects & 182 & $2.46 \pm 0.52$ & $2.45 \pm 0.46$ & $2.84 \pm 0.38^{\mathrm{a}}$ \\
Men & 91 & $2.37 \pm 0.51$ & $2.34 \pm 0.47$ & $2.96 \pm 0.45^{\mathrm{a}}$ \\
Women & 91 & $2.56 \pm 0.51$ & $2.55 \pm 0.43$ & $2.71 \pm 0.24$ \\
Smokers & 41 & $2.42 \pm 0.45$ & $2.37 \pm 0.49$ & $3.04 \pm 0.39^{\mathrm{a}}$ \\
Non-smokers & 140 & $2.47 \pm 0.54$ & $2.46 \pm 0.45$ & $2.73 \pm 0.35$ \\
Type II diabetic & 264 & $3.22 \pm 0.95$ & $3.32 \pm 0.81$ & $3.57 \pm 1.13$ \\
Men & 130 & $3.03 \pm 0.76$ & $3.25 \pm 0.91$ & $3.46 \pm 1.01$ \\
Women & 134 & $3.39 \pm 1.08$ & $3.39 \pm 0.71$ & $3.68 \pm 1.27$ \\
\hline
\end{tabular}

${ }^{\mathrm{a}} p<0.05$ vs $G G$ or $G A$

\section{Results}

The allele frequency of the $A$ allele was $27 \%$ in both control subjects and diabetic patients. Among control subjects the $A A$ genotype, present in $6 \%$, was associated with higher fibrinogen concentrations than other genotypes (Table $1 ; p<0.03$ vs $G G$ or $G A$ ). The $G / A$ 455 genotype effect was significant among men $(p<0.03)$ and smokers $(p<0.05)$, but was smaller and non-significant among women and non-smokers. Among the patients with Type II diabetes, the presence of the $A$ allele appeared to be associated with higher fibrinogen concentrations, but the differences among the three genotypes were not statistically significant.

Plasma fibrinogen was higher in women (controls: $2.6 \pm 0.5$ vs $2.4 \pm 0.5 \mathrm{~g} / 1$ in men, $p<0.03$; patients: $3.4 \pm 1.0$ vs $3.1 \pm 0.8 \mathrm{~g} / \mathrm{l}$ in men, $p<0.02$ ). There was no significant difference in plasma fibrinogen concentration between smokers and non-smokers in control subjects or patients. In stepwise multiple linear regression, age, sex and the $A A$ genotype contributed $5.6,3.9$ and $4 \%$, respectively to the variance in plasma fibrinogen in control subjects.

Despite similar age, sex and genotype distributions, patients with Type II diabetes had higher fibrinogen concentrations $(3.3 \pm 0.9$ vs $2.5 \pm 0.5 \mathrm{~g} / \mathrm{l}$ in control subjects, $p<0.0001)$. They also had higher triglyceride and lower HDL-cholesterol concentrations $(p<0.0001)$ and higher BMI $(p<0.05)$. Over half of them had microalbuminuria or albuminuria. In multivariate analysis, only MAER, age, serum creatinine, sex and apolipoprotein A1 were statistically significant determinants of plasma fibrinogen, contributing $14.4,5.9,5,2.9$ and $3.5 \%$ respectively to the variance in diabetic patients.

The differences in clinical, biochemical and genetic variables between patients with and without ischaemic heart disease are summarised in Table 2. Patients with ischaemic heart disease had higher plasma fibrinogen concentrations $(p<0.05)$. A relation was found between the $G / A-455$ genotype and ischaemic heart disease $(p<0.005$, Table 2$)$ with the $A A$ genotype present in $16 \%$ in those with ischaemic heart 
Table 2. Fibrinogen genotype, plasma fibrinogen concentration and other clinical characteristics in diabetic patients with and without ischaemic heart disease

\begin{tabular}{lcc}
\hline & IHD $(n=55)$ & No IHD $(n=209)$ \\
\hline Fibrinogen genotype & $33: 13: 9^{\mathrm{b}}$ & $111: 87: 11$ \\
GG : GA : AA & $(60: 24: 16 \%)$ & $(53: 42: 5 \%)$ \\
Fibrinogen (g/l) & $3.5 \pm 0.9^{\mathrm{a}}$ & $3.2 \pm 0.9$ \\
Age (year) & $62.8 \pm 12.0^{\mathrm{c}}$ & $49.7 \pm 16.1$ \\
Mean arterial pressure & $104.5 \pm 11.8^{\mathrm{c}}$ & $98.2 \pm 11.8$ \\
(mm) & $12.8 \pm 8.2^{\mathrm{a}}$ & $10.0 \pm 8.0$ \\
Diabetes duration (year) & $8.1 \pm 2.3^{\mathrm{a}}$ & $7.3 \pm 2.2$ \\
HbA $_{1 \mathrm{c}}(\%)$ & $3.5 \pm 1.0^{\mathrm{a}}$ & $3.2 \pm 0.9$ \\
LDL-cholesterol (mmol/l) $^{\mathrm{a}}$ & $1.4 \pm 0.3^{\mathrm{b}}$ & $1.2 \pm 0.3$ \\
Apo B (mg/dl) &
\end{tabular}

IHD, ischaemic heart disease; ${ }^{\mathrm{a}} p<0.05 ;{ }^{\mathrm{b}} p<0.005$; ${ }^{c} p<0.0001$ vs number of ischaemic heart disease (IHD) patients

disease (vs $5 \%$ in non-affected patients, $p<0.005$; $p<0.02$ if corrected for the number of genotypes being compared). In a multiple logistic regression model the $A A$ genotype was also an independent risk factor of ischaemic heart disease [odds ratio (OR) 4.19, $95 \%$ confidence intervals $1.39-12.58, p<0.01]$ in diabetic patients, together with age [OR 1.05 (1.03-1.08), $p<0.001)$, and mean arterial blood pressure [OR $1.03(1.00-1.06), p<0.03)$.

\section{Discussion}

In this study, we have shown that the $\beta$-fibrinogen gene $G / A-455$ polymorphism contributes to $4 \%$ of the variance in plasma fibrinogen concentration in Chinese non-diabetic subjects. In Europeans, its contribution ranges from $1 \%$ [5] to $3.6 \%$ [4]. Subjects with the $A A$ genotype in this study had higher fibrinogen concentrations than those with the $G G$ or $G A$ genotypes, similar to findings in Europeans [3-7]. The allele frequency of the less frequent $A$ allele was $27 \%$, comparable with the frequencies of $21 \%$ [3] and $27 \%$ [5] reported for Europeans.

It is well known that genetic and environmental factors interact in determining plasma concentrations of haemostatic factors $[7,8]$. In this study, the effect of the $A A$ genotype was strongest among male control subjects, in keeping with a gender-genotype interaction [8]. The higher fibrinogen concentrations in women has been attributed to environmental influences such as the menopause or oral contraceptives [7]. An interaction between the $G / A-455$ genotype and smoking has also been reported $[3,5,7]$. In general, the genotype effect appears to be stronger among smokers $[3,5]$ as in this study, with the highest fibrinogen concentrations seen in smokers with $A A$ genotype. The $A-455$ sequence change is in complete allel- ic association in Caucasians, as well as in Hong Kong Chinese (O.C.K. Ma et al, unpublished observations) with a $C-148 T$ change [9] located close to the consensus interleukin- 6 response element and may therefore be linked to increased fibrinogen gene transcription in response to interleukin-6 released from lung macrophages following tissue damage induced by smoking. A genotype-environment interaction was also apparent in the diabetic patients in whom the genotype effect was much smaller, probably because of the predominant effect of nephropathy, the most important factor contributing to their increased fibrinogen concentrations.

In this study the $A A$ genotype, found in increased frequency in German subjects with large vessel cerebral artery disease [5], was also associated with ischaemic heart disease in Chinese Type II diabetic patients. Together with the association of the $A$ allele with the severity of coronary artery disease in non-diabetic Caucasians [3] and with ischaemic stroke in Japanese [10], this provided further support for the association of the $A$ allele with a genetic predisposition to atherosclerosis. We were not able to show a gene dosage effect of the $A$ allele on either plasma fibrinogen concentration or risk of ischaemic heart disease in the heterozygous subjects. The risk of ischaemic heart disease even seemed to be low in the heterozygous patients. This could possibly be explained by the multiple environmental factors contributing to changes in plasma fibrinogen concentration and the risk of ischaemic heart disease, so that the genetic effect would only be clearly shown in the homozygous state. In the German study, a gene dosage effect on the risk of cerebral atherosclerosis was also not shown [5].

In conclusion, our findings suggest that the $G / A$ 455 polymorphism of the $\beta$-fibrinogen gene is a genetic determinant of plasma fibrinogen concentrations and ischaemic heart disease in this Chinese cohort. It also interacts with environmental influences associated with smoking, the female sex and Type II diabetes in determining the concentration of plasma fibrinogen.

Acknowledgements. We thank Mr S. Yeung for assistance with data processing. This study was supported by a grant from the Committee on Research and Conference Grants, the University of Hong Kong.

\section{References}

1. Ostermann H, van de Loo J (1986) Factors of the hemostatic system in diabetic patients. A survey of controlled studies. Haemostasis 16: 384-416

2. Ernst E (1993) The role of fibrinogen as a cardiovascular risk factor. Atherosclerosis 100: 1-12

3. Behague I, Poirier O, Nicaud V et al. (1996) $\beta$-Fibrinogen gene polymorphisms are associated with plasma fibrinogen 
and coronary artery disease in patients with myocardial infarction. The ECTIM study. Circulation 93: 440-449

4. Thomas AE, Green F, Lamlum H, Humphries S (1995) The association of combined alpha and beta fibrinogen genotype on plasma fibrinogen levels in smokers and non-smokers. J Med Genet 32: 585-589

5. Kessler C, Spitzer C, Stauske D et al. (1997) The apolipoprotein $\mathrm{E}$ and $\beta$-fibrinogen $\mathrm{G} / \mathrm{A}-455$ gene polymorphisms are associated with ischemic stroke involving large-vessel disease. Arterioscler Thromb Vasc Biol 17: 2880-2884

6. Carter AM, Mansfield MW, Stickland MH, Grant PJ (1996) $\beta$-fibrinogen gene-455 G/A polymorphism and fibrinogen concentrations: risk factors for coronary artery disease in subjects with NIDDM. Diabetes Care 19: 1265-1268

7. Humphries SE, Ye S, Talmud P et al. (1995) European Atherosclerosis Research Study: genotype at the fibrinogen locus $\left(\mathrm{G}_{-455}\right.$ - $\mathrm{A} \beta$-gene $)$ is asssociated with differences in plasma fibrinogen levels in young men and women from differ- ent regions in Europe: evidence for gender-genotype-environment interaction. Arterioscler Thromb Vasc Biol 15: 96-104

8. Lam KSL, Ma OCK, Bourke C, Chan LC, Janus ED (1998) Genetic influence of the R/Q353 genotype on factor VII activity is overwhelmed by environmental factors in Chinese patients with Type II (non-insulin-dependent) diabetes mellitus. Diabetologia 41: 760-766

9. Thomas A, Lamlum H, Humphries S, Green F (1994) Linkage disequilibrium across the fibrinogen locus as shown by five genetic polymorphisms, G/ $\mathrm{A}^{-455}$ (HaeIII), $\mathrm{C} / \mathrm{T}^{-148} \mathrm{Hin}-$ dIII/AluI), T/G ${ }^{-1689}$ (AvaII) and Bcl1 ( $\beta$-fibrinogen) and Taq1 (a-fibrinogen), and their detection by PCR. Hum Mutat 3: 79-81

10. Nishiuma S, Kario K, Yakushijin K et al. (1998) Genetic variation in the promotor region of the beta-fibrinogen gene is associated with ischemic stroke in a Japanese population. Blood Coagul Fibrinolysis 9: 373-379 